\title{
ACCURACY OF VARIOUS APPROXIMATIONS TO EXCHANGE AND CORRELATION FOR THE ELECTRON DENSITY DISTRIBUTION IN ATOMS AND SMALL MOLECULES it
}

\author{
M.P.C.M. KRIJN and D. FEIL \\ Chemical Physics Laboratory, Twente University of Technology, P.O. Box 217, 7500 AE Enschede, The Netherlands \\ Received 16 March 1988; in final form 21 June 1988
}

\begin{abstract}
The general usefulness of various local and non-local approximations to the exchange-correlation potential in density functional theory is studied by comparing resulting electron density distributions to essentially exact results for light atoms. The correlation contribution to the electron density in $\mathrm{CO}$ and $\mathrm{H}_{2} \mathrm{O}$ is compared with $\mathrm{CI}$ results. It is concluded that density functional theory provides a viable alternative to $\mathrm{HF}$ and $\mathrm{CI}$ approaches for the calculation of deformation densities, although the response of the electron density to the correlation potential is only moderately accurate.
\end{abstract}

\section{Introduction}

Much of our understanding of chemical bonding is based on the electron density distribution [1-3], the fundamental variable to describe the ground state of a system in an external potential according to density functional theory (DFT) [4]. DFT has become an indispensable tool in calculating the ground-state electronic properties of solids [5]. Although equally applicable to finite systems, DFT has not yet superseded Hartree-Fock (HF) and the computationally more demanding configuration interaction (CI) approaches.

In DFT, the many-body problem of $N$ electrons moving in a local external potential is reformulated in terms of a single-particle equation describing an electron moving in the mean field of the remaining $N-1$ electrons [6]. The many-body nature enters via the complexity of the mean field, for which some approximation must be adopted. The simplest, and most often used, is the local density (LD) approximation, justified only in the limit of slowly varying densities, but nevertheless yielding surprisingly accurate results for atoms and molecules, systems with a rapidly varying density [7-9]. Important contri-

t Supported in part by the Netherlands Foundation for Chemical Research (SON) with financial aid from the Netherlands Organization for Advancement of Pure Research (ZWO). butions improving on the LD approximation have recently been made by Langreth, Perdew, Mehl, and Hu [10-13]. However, discrepancies with CI results and experiment leave room for further refinements.

The aim of the present paper is to investigate whether DFT provides a viable alternative to $\mathrm{HF}$ and CI methods for calculating, for atoms and molecules, the electron density distribution and properties derived from it. To test the domain of validity of various local and non-local approximations to exchange and correlation in DFT, results are compared with those obtained from accurate uncorrelated (HF) and correlated (CI) wavefunctions, available only for a limited selection of atoms and small molecules. Studied here are the ground-state electron density distributions of the closed-shell systems $\mathrm{He}, \mathrm{Li}^{+}$, $\mathrm{Be}^{2+}, \mathrm{Be}, \mathrm{Ne}, \mathrm{Na}^{+}, \mathrm{Mg}^{2+}, \mathrm{Mg}, \mathrm{Ar}, \mathrm{K}^{+}, \mathrm{Ca}^{2+}, \mathrm{CO}$, and $\mathrm{H}_{2} \mathrm{O}$.

\section{Methods}

\subsection{Exchange-correlation potentials}

Applying the variation principle to the total electronic energy of a molecular containing $N$ electrons and, in the spin-restricted case, given by 


$$
\begin{aligned}
E & =\frac{1}{2} \sum_{i=1}^{N} \int\left|\nabla \phi_{i}\right|^{2} \mathrm{~d}^{3} r+\int \rho v_{\mathrm{ext}} \mathrm{d}^{3} r \\
& +\frac{1}{2} \iint \frac{\rho(\boldsymbol{r}) \rho\left(\boldsymbol{r}^{\prime}\right)}{\left|\boldsymbol{r}-\boldsymbol{r}^{\prime}\right|} \mathrm{d}^{3} \boldsymbol{r} \mathrm{d}^{3} \boldsymbol{r}^{\prime}+E_{\mathrm{xc}}[\rho]
\end{aligned}
$$

leads to the set of coupled Schrödinger-type oneelectron equations

$\left(-\frac{1}{2} \nabla^{2}+v_{\mathrm{ext}}+\int \frac{\rho\left(\boldsymbol{r}^{\prime}\right)}{\left|\boldsymbol{r}-\boldsymbol{r}^{\prime}\right|} \mathrm{d}^{3} \boldsymbol{r}^{\prime}+v_{\mathrm{XC}}\right) \phi_{i}=\epsilon_{i} \phi_{i}$,

where

$\rho=\sum_{i=1}^{N}\left|\phi_{i}\right|^{2}$

is the electron density distribution, $v_{\text {ext }}$ the Coulomb potential produced by the atomic nuclei, and

$v_{\mathrm{XC}}=\frac{\delta E_{\mathrm{XC}}[\rho]}{\delta p}$

the exchange-correlation potential. In the LD approximation, the exchange-correlation energy $E_{\mathrm{XC}}$ is approximated by

$E_{\mathrm{Xc}}[\rho]=\int \rho \epsilon_{\mathrm{Xc}}(\rho) \mathrm{d}^{3} r$,

where $\epsilon_{X C}$ is the exchange-correlation energy per particle of the homogeneous electron gas, resulting in an exchange-correlation potential

$v_{\mathrm{XC}}=\frac{\partial}{\partial \rho}\left[\rho \epsilon_{\mathrm{XC}}(\rho)\right]$.

Consequently, the LD approximation is uniquely defined in terms of the exact but imprecisely known properties of the homogeneous electron gas. Within this approximation, the exchange part $v_{x}$ of $v_{x c}$ leads to the simple expression arrived at by Kohn and Sham

$v_{\mathrm{X}}=-3 \alpha(3 \rho / 8 \pi)^{1 / 3}$

with $\alpha=2 / 3$ [6]. An accurate local expression for the correlation part $v_{\mathrm{C}}$ of $v_{\mathrm{xc}}$ has been obtained by Vosko et al. $[14,15]$ from a parametrization of the Monte Carlo results of Ceperly and Alder [16] for the homogeneous electron gas, believed to be in close agrecment with the exact results.

Apart from errors inherent in assuming a local character, the exchange energy only partially corrects for self-interaction errors, resulting in an exponential decay of the exchange potential instead of the $-1 / r$ behaviour at large distances from the nuclei of neutral atoms. So far, correction procedures have proved to be either unsatisfactory or orbital dependent, an undesired property. The correlation energy is also affected by self-correlation errors. In molecular systems, the correlation energy of like-spin electron pairs is small compared to that of opposite-spin electron pairs. In the homogeneous electron gas, however, the like-spin and opposite-spin pairs contribute almost equally to the correlation energy. Therefore, in molecular systems, correlation energies have to be corrected by subtracting the like-spin contribution. These arguments led Stoll et al. [17,18] to the following expression for the correlation potential in the spin-restricted case:

$v_{\mathrm{C}}=\frac{\partial}{\partial \rho}\left[\rho \epsilon_{\mathrm{C}}(\rho)-\rho \epsilon_{\mathrm{C}}\left(\frac{1}{2} \rho\right)\right]$

thereby retaining its local character.

\subsection{Computational details}

For atoms, results are obtained by numerically solving eq. (2). In the calculations on $\mathrm{CO}$ and $\mathrm{H}_{2} \mathrm{O}$, the discrete variational method (DVM), as proposed by Ellis and Painter [19] and developed by Baerends et al. [20,21], is employed. Following their method, the orbitals $\phi_{i}$ are expanded in terms of Slater-type orbital (STO) functions, using GaussLegendre quadrature [22] to calculate the matrix elements of the secular equation. To reduce the computational effort, while retaining the desired accuracy, Coulomb and exchange-correlation potentials are evaluated from an expansion of the density $\rho$ in one-centre STO fit functions

$\tilde{\rho}=\sum_{i} a_{i} f_{i}$.

The expansion coefficients $a_{i}$ are determined by minimizing the discrepancy index

$D=\int(\rho-\tilde{\rho})^{2} \mathrm{~d}^{3} r$

subject to the constraint $\int \tilde{\rho} \mathrm{d}^{3} r=N$. A quadruple-zeta and triple-zcta basis set of STOs, augmented with dand f-type polarization functions, are used in the case of $\mathrm{CO}$ and $\mathrm{H}_{2} \mathrm{O}$, respectively. The exponents used 
are reported in ref. [23] and ref. [24] for $\mathrm{CO}$ and $\mathrm{H}_{2} \mathrm{O}$, respectively. Results obtained this way are assumed not to deviate significantly from fully numerical results, a statement supported in ref. [23].

\section{Results}

\subsection{Introduction}

Among others, Gunnarsson and Jones [25], Painter [26], Perdew and Zunger [27], Langreth and Mehl [12], and Becke [28] realized that the accuracy of $\mathrm{LD}$ approximations is primarily limited by approximations made to the exchange part of the exchange-correlation energy functional. For atoms, LD approximations underestimate exchange energies by around $10 \%$, whereas correlation energies are overestimated by $100 \%$. However, correcting correlation energies for self-correlation by subtracting the likespin contribution, as proposed by Stoll et al. [17,18], markedly improves the agreement with exact results, leaving a discrepancy of about $10 \%$. Therefore, since exchange energies outweigh correlation energies by an order of magnitude, efforts to improve on the LD approximation should focus primarily on the exchange contribution.

Non-local exchange-only functionals, reported as being a considerable improvement on LD exchange energies, are taken from Langreth and Mehl (hereafter denoted as $\mathrm{LM}_{\mathbf{X}}$ ) [12], based on a formulation in reciprocal space, and Becke $\left(B_{X}\right)[28]$, arrived at from a semi-empirical approach. Both functionals are known to yield energies in close agreement with the corresponding HF values, when calculated from the HF density. However, since density functionals, in general, are not constructed in a variational form, there is no guarantee that a variationally derived energy is an upper bound to the true HF exchange energy. Consequently, an electron density distribution determined variationally may not produce values for the exchange energy in close agreement with the exact values. The same applies to the correlation energy.

Here we present the results of variationally determined electron density distributions, obtained using the local Kohn-Sham exchange-only potential $\left(\mathbf{K S}_{\mathbf{X}}\right.$, eq. (7) ) [6] and the abovementioned non-local potentials $\mathrm{LM}_{\mathbf{X}}$ and $\mathrm{B}_{\mathbf{X}}$. Results are compared with electron density distributions obtained from HF wavefunctions. In subsequent calculations, the effect of correlation is taken into account by adding the local correlation potential of Vosko et al. $\left(\mathrm{VWN}_{\mathrm{C}}\right.$ ) [14,15], corrected for self-correlation according to the method of Stoll et al., to the exchange-only potentials $\mathbf{K S}_{\mathbf{X}}, \mathbf{L M}_{\mathbf{X}}$, and $\mathbf{B}_{\mathbf{X}}$. Results are compared with those obtained from $\mathrm{CI}$ wavefunctions, available for $\mathrm{He}, \mathrm{Li}^{+}, \mathrm{Be}^{2+}, \mathrm{Be}$, and $\mathrm{Ne}$.

Unified approaches to exchange and correlation yielded the well-known local $\mathrm{X} \alpha$ potential (eq. (7), with $\alpha$ an adjustable parameter, taken to be 0.7 throughout the present work) and, recently, the nonlocal exchange-correlation potential of Langreth and Mehl ( $\left.\mathrm{LM}_{\mathrm{XC}}\right)$ [12]. Results obtained so far strongly support the superiority of $\mathrm{LM}_{\mathrm{XC}}$ to $\mathrm{X} \alpha$ results, or $\mathrm{LD}$ results in general. Improved $\mathrm{LM}_{\mathrm{XC}}$ results for the electron density distribution compared to those of the LD approximation are briefly discussed.

The physical interpretation of the eigenvalues $\epsilon_{i}$ and orbitals $\phi_{i}$ in eq. (2) remains unclear. Recently, however, Almbladh and von Barth [29] demonstrated that the highest occupied orbital eigenvalue, obtained with the exact (although unknown) density functional, equals the exact ionization energy, even for systems of finite size. For this reason, we also compare orbital eigenvalues with experiment and supposedly exact values.

Finally, the response of the electron density distribution to the inclusion of correlation effects in the exchange-correlation potential is discussed for $\mathrm{He}$, $\mathrm{Be}, \mathrm{Ne}, \mathrm{CO}$, and $\mathrm{H}_{2} \mathrm{O}$ respectively.

\subsection{Atoms}

To facilitate a quantitative comparison between DFT, HF, and supposedly exact results, the lower moments of the electron density, defined as $\left\langle r^{n}\right\rangle=$ $\int r^{n} \rho(r) \mathrm{d}^{3} r$, are calculated for the various exchangecorrelation potentials discussed, and summarized in table 1 for the closed-shell systems $\mathrm{He}, \mathrm{Li}^{+}, \mathrm{Be}^{2+}$, $\mathrm{Be}, \mathrm{Ne}, \mathrm{Na}^{+}, \mathrm{Mg}^{2+}, \mathrm{Mg}, \mathrm{Ar}, \mathrm{K}^{+}$, and $\mathrm{Ca}^{2+}$. Also shown are the electron densities at the nuclei and eigenvalues of the highest occupied orbitals. Electron densities including correlation contributions, referred to as the exact density, are available for $\mathrm{He}$, $\mathrm{Li}^{+}, \mathrm{Be}^{2+}, \mathrm{Be}$, and $\mathrm{Ne}$. For $\mathrm{He}, \mathrm{Li}^{+}$, and $\mathrm{Be}^{2+}$, these were obtained from Hylleraas-type wavefunctions 
Table 1

Charge densities at the nuclei, moments and highest occupied atomic orbital eigenvalues for $\mathrm{He}, \mathrm{Li}^{+}, \mathrm{Be}^{2+}, \mathrm{Be}, \mathrm{Ne}, \mathrm{Na}{ }^{+}, \mathrm{Mg}^{2+}, \mathrm{Mg}^{\mathrm{Ar}} \mathrm{Ar}$ $\mathrm{K}^{+}$, and $\mathrm{Ca}^{2+}$. All in atomic units

\begin{tabular}{|c|c|c|c|c|c|c|c|}
\hline Atom & & $\rho(0)$ & $\left\langle r^{-2}\right\rangle$ & $\left\langle r^{-1}\right\rangle$ & $\langle r\rangle$ & $\left\langle r^{2}\right\rangle$ & $-\epsilon_{\mathrm{HOAO}}$ \\
\hline \multirow[t]{10}{*}{$\mathrm{He}$} & $\times 0.7$ & 3.53 & 11.72 & 3.31 & 1.923 & 2.59 & 0.54 \\
\hline & $\mathrm{LM}_{\mathbf{x C}}$ & 3.60 & 11.92 & 3.35 & 1.886 & 2.47 & 0.58 \\
\hline & $\mathrm{KS}_{\mathrm{x}}$ & 3.47 & 11.56 & 3.28 & 1.944 & 2.66 & 0.52 \\
\hline & $\mathrm{LM}_{\mathbf{X}}$ & 3.66 & 12.02 & 3.36 & 1.892 & 2.50 & 0.54 \\
\hline & $\mathbf{B}_{\mathbf{X}}$ & 3.84 & 12.44 & 3.42 & 1.855 & 2.41 & 0.51 \\
\hline & $\mathrm{KS}_{\mathrm{X}}+\mathrm{VWN}_{\mathrm{C}}$ & 3.50 & 11.64 & 3.30 & 1.931 & 2.61 & 0.55 \\
\hline & $\mathrm{LM}_{\mathrm{X}}+\mathrm{VWN}_{\mathrm{C}}$ & 3.69 & 12.10 & 3.37 & 1.879 & 2.46 & 0.57 \\
\hline & $\mathrm{B}_{\mathrm{X}}+\mathrm{VWN}_{\mathrm{C}}$ & 3.86 & 12.52 & 3.43 & 1.843 & 2.37 & 0.54 \\
\hline & $\mathrm{HF}^{\mathrm{a})}$ & 3.60 & 11.99 & 3.38 & 1.855 & 2.37 & 0.92 \\
\hline & exact $\left.^{b}\right)$ & 3.63 & 12.03 & 3.38 & 1.858 & 2.39 & $0.90^{f i}$ \\
\hline \multirow[t]{10}{*}{$\mathrm{Li}^{+}$} & $\mathrm{X} 0.7$ & 13.49 & 29.4 & 5.31 & 1.168 & 0.935 & 2.16 \\
\hline & $\mathbf{L M}_{\mathbf{X C}}$ & 13.67 & 29.7 & 5.35 & 1.157 & 0.913 & 2.21 \\
\hline & $\mathbf{K} \mathbf{S}_{\mathbf{X}}$ & 13.35 & 29.1 & 5.29 & 1.175 & 0.947 & 2.12 \\
\hline & $\mathrm{LM}_{\mathbf{X}}$ & 13.80 & 29.8 & 5.36 & 1.157 & 0.914 & 2.17 \\
\hline & $\mathbf{B}_{\mathbf{x}}$ & 14.24 & 30.5 & 5.43 & 1.141 & 0.892 & 2.14 \\
\hline & $\mathrm{KS}_{\mathrm{x}}+\mathrm{VWN}_{\mathrm{C}}$ & 13.39 & 29.2 & 5.30 & 1.172 & 0.942 & 2.16 \\
\hline & $\mathbf{L M}_{\mathbf{X}}+\mathrm{VWN}_{C}$ & 13.85 & 29.9 & 5.37 & 1.153 & 0.909 & 2.21 \\
\hline & $\mathrm{B}_{\mathrm{X}}+\mathrm{VWN}_{\mathrm{C}}$ & 14.29 & 30.6 & 5.43 & 1.139 & 0.887 & 2.18 \\
\hline & $H^{c)}$ & 13.68 & 29.8 & 5.38 & 1.145 & 0.891 & 2.79 \\
\hline & exact $^{\text {b) }}$ & 13.71 & 29.9 & 5.38 & 1.146 & 0.893 & $2.78^{f)}$ \\
\hline \multirow[t]{10}{*}{$\mathrm{Be}^{2+}$} & $\times 0.7$ & 33.99 & 55.0 & 7.31 & 0.840 & 0.479 & 4.78 \\
\hline & $\mathbf{L M}_{\mathbf{X C}}$ & 34.34 & 55.4 & 7.35 & 0.835 & 0.472 & 4.84 \\
\hline & $\mathrm{KS}_{\mathrm{X}}$ & 33.73 & 54.7 & 7.29 & 0.844 & 0.484 & 4.73 \\
\hline & $\mathrm{LM}_{\mathbf{x}}$ & 34.57 & 55.6 & 7.36 & 0.834 & 0.472 & 4.80 \\
\hline & $\mathrm{B}_{\mathrm{X}}$ & 35.39 & 56.6 & 7.43 & 0.826 & 0.463 & 4.77 \\
\hline & $\mathbf{K S}_{\mathrm{x}}+\mathbf{V W N}_{\mathrm{C}}$ & 33.79 & 54.7 & 7.29 & 0.843 & 0.482 & 4.77 \\
\hline & $\mathrm{LM}_{\mathrm{x}}+\mathrm{VWN}_{\mathrm{C}}$ & 34.64 & 55.7 & 7.36 & 0.833 & 0.470 & 4.85 \\
\hline & $\mathbf{B}_{\mathrm{x}}+\mathrm{VWN}_{\mathrm{c}}$ & 35.46 & 56.7 & 7.43 & 0.825 & 0.462 & 4.81 \\
\hline & $H F^{c)}$ & 34.41 & 55.6 & 7.38 & 0.828 & 0.464 & 5.67 \\
\hline & exact $^{b)}$ & 34.40 & 55.7 & 7.38 & 0.828 & 0.464 & $5.66^{f)}$ \\
\hline \multirow[t]{10}{*}{$\mathrm{Be}$} & $\mathrm{X} 0.7$ & 34.98 & 56.90 & 8.34 & 6.15 & 17.51 & 0.18 \\
\hline & $\mathrm{LM}_{\mathrm{XC}}$ & 35.35 & 57.35 & 8.38 & 6.05 & 16.59 & 0.22 \\
\hline & $\mathbf{K} \mathbf{S}_{\mathrm{x}}$ & 34,70 & 56.53 & 8.31 & 6.22 & 17.92 & 0.17 \\
\hline & $\mathrm{LM}_{\mathbf{x}}$ & 35.59 & 57.57 & 8.38 & 6.14 & 17.28 & 0.18 \\
\hline & $\mathbf{B}_{\mathbf{x}}$ & 36.30 & 58.40 & 8.46 & 6.02 & 16.54 & 0.16 \\
\hline & $\mathrm{KS}_{\mathbf{x}}+\mathrm{VWM}_{\mathrm{C}}$ & 34.78 & 56.65 & 8.32 & 6.15 & 17.42 & 0.19 \\
\hline & $\mathrm{LM}_{\mathrm{X}}+\mathrm{VWM}_{\mathrm{C}}$ & 35.67 & 57.68 & 8.40 & 6.07 & 16.81 & 0.20 \\
\hline & $\mathrm{B}_{\mathrm{X}}+\mathrm{VWN}_{\mathrm{C}}$ & 36.39 & 58.52 & 8.47 & 5.96 & 16.11 & 0.18 \\
\hline & $H F^{\text {a) }}$ & 35.43 & 57.61 & 8.41 & 6.13 & 17.32 & 0.31 \\
\hline & exact ${ }^{\text {d) }}$ & 35.37 & 57.60 & 8.43 & 5.98 & 16.26 & $0.34^{f)}$ \\
\hline \multirow[t]{10}{*}{$\mathrm{Ne}$} & $\mathrm{X} 0.7$ & 616.0 & 412.5 & 31.04 & 8.01 & 9.86 & 0.47 \\
\hline & $\mathbf{L M}_{\mathrm{XC}}$ & 618.8 & 413.6 & 31.05 & 7.98 & 9.70 & 0.50 \\
\hline & $\mathrm{KS}_{\mathbf{X}}$ & 614.2 & 411.4 & 30.95 & 8.07 & 10.04 & 0.44 \\
\hline & $\mathrm{LM}_{\mathrm{x}}$ & 620.4 & 414.2 & 31.05 & 8.02 & 9.85 & 0.44 \\
\hline & $\mathbf{B}_{\mathbf{X}}$ & 624.3 & 416.0 & 31.12 & 7.98 & 9.74 & 0.41 \\
\hline & $\mathrm{KS}_{\mathrm{x}}+\mathrm{VWN}_{\mathrm{c}}$ & 614.4 & 411.5 & 30.98 & 8.04 & 9.94 & 0.47 \\
\hline & $\mathrm{LM}_{\mathrm{X}}+\mathrm{VWN}_{\mathrm{C}}$ & 620.6 & 414.4 & 31.07 & 7.99 & 9.75 & 0.47 \\
\hline & $B_{X}+V W N_{C}$ & 624.5 & 416.1 & 31.14 & 7.95 & 9.65 & 0.44 \\
\hline & $\mathrm{HF}^{\mathrm{a})}$ & 620.15 & 414.66 & 31.11 & 7.89 & 9.37 & 0.85 \\
\hline & exact ${ }^{e)}$ & 620.20 & 414.72 & 31.11 & 7.94 & 9.55 & $0.79^{f)}$ \\
\hline
\end{tabular}


Table 1 (continued)

\begin{tabular}{|c|c|c|c|c|c|c|c|}
\hline Atom & & $\rho(0)$ & $\left\langle r^{-2}\right\rangle$ & $\left\langle r^{-1}\right\rangle$ & $\langle r\rangle$ & $\left\langle r^{2}\right\rangle$ & $-\boldsymbol{\epsilon}_{\text {HOAO }}$ \\
\hline \multirow[t]{4}{*}{$\mathrm{Na}^{+}$} & $\mathrm{KS}_{\mathbf{x}}$ & 825.9 & 505.1 & 34.99 & 6.71 & 6.67 & 1.28 \\
\hline & $\mathrm{LM}_{\mathrm{X}}$ & 833.5 & 508.2 & 35.09 & 6.68 & 6.59 & 1.28 \\
\hline & $\mathrm{B}_{\mathbf{X}}$ & 838.2 & 510.2 & 35.16 & 6.65 & 6.52 & 1.25 \\
\hline & $H F^{c}$ & 833.4 & 508.8 & 35.14 & 6.62 & 6.41 & 1.80 \\
\hline \multirow[t]{4}{*}{$\mathrm{Mg}^{2+}$} & $K S_{X}$ & 1082.3 & 608.8 & 39.02 & 5.78 & 4.85 & 2.39 \\
\hline & $\mathrm{LM}_{x}$ & 1091.3 & 612.2 & 39.11 & 5.76 & 4.81 & 2.39 \\
\hline & $\mathrm{B}_{\mathrm{X}}$ & 1097.0 & 614.4 & 39.19 & 5.74 & 4.76 & 2.36 \\
\hline & $\mathrm{HF}^{\mathrm{c})}$ & 1091.3 & 613.0 & 39.16 & 5.73 & 4.72 & 3.01 \\
\hline \multirow[t]{4}{*}{$\mathrm{Mg}$} & $\mathrm{KS}_{\mathrm{x}}$ & 1085.1 & 610.4 & 39.78 & 12.27 & 29.49 & 0.14 \\
\hline & $\mathrm{LM}_{\mathrm{x}}$ & 1093.9 & 613.7 & 39.86 & 12.28 & 29.40 & 0.14 \\
\hline & $\mathrm{B}_{\mathrm{x}}$ & 1099.5 & 615.8 & 39.93 & 12.18 & 28.73 & 0.13 \\
\hline & $H F^{a)}$ & 1093.7 & 614.4 & 39.92 & 12.26 & 29.61 & 0.25 \\
\hline \multirow[t]{4}{*}{$\mathrm{Ar}$} & $K S_{x}$ & 3818.6 & 1457.0 & 69.57 & 16.21 & 26.80 & 0.33 \\
\hline & $\mathrm{LM}_{\mathrm{X}}$ & 3838.9 & 1462.0 & 69.67 & 16.13 & 26.37 & 0.33 \\
\hline & $\mathbf{B}_{\mathrm{X}}$ & 3851.3 & 1465.1 & 69.74 & 16.05 & 26.03 & 0.31 \\
\hline & $\mathrm{HF}^{\text {a) }}$ & 3840.2 & 1463.5 & 69.73 & 16.07 & 26.04 & 0.59 \\
\hline \multirow[t]{4}{*}{$\mathrm{K}^{+}$} & $\mathrm{KS}_{\mathbf{x}}$ & 4513.7 & 1635.1 & 74.52 & 14.23 & 19.82 & 0.86 \\
\hline & $\mathbf{L M}_{\mathbf{x}}$ & 4536.5 & 1640.5 & 74.62 & 14.19 & 19.61 & 0.86 \\
\hline & $\mathrm{B}_{\mathrm{X}}$ & 4550.4 & 1643.7 & 74.70 & 14.13 & 19.39 & 0.85 \\
\hline & $H F^{c)}$ & 4539.9 & 1642.3 & 74.67 & 14.18 & 19.57 & 1.17 \\
\hline \multirow[t]{4}{*}{$\mathrm{Ca}^{2+}$} & $\mathrm{KS}_{\mathbf{X}}$ & 5289.6 & 1823.9 & 79.45 & 12.78 & 15.56 & 1.52 \\
\hline & $\mathbf{L M}_{\mathbf{X}}$ & 5314.8 & 1829.6 & 79.55 & 12.75 & 15.43 & 1.53 \\
\hline & $\mathbf{B}_{\mathrm{X}}$ & 5330.2 & 1833.0 & 79.63 & 12.70 & 15.28 & 1.51 \\
\hline & $\mathrm{HF}^{\mathrm{c}}$ & 5319.2 & 1831.5 & 79.60 & 12.75 & 15.46 & 1.88 \\
\hline
\end{tabular}

s) Combined results of ref. [30] and those corresponding to the wavefunctions reported in ref. [31].

b) Results as quoted in refs. [12,32]. ' Results corresponding to the wavefunctions reported in ref. [31 ].

d) $\mathrm{CI}$ results [33]. "CI results [34]. ${ }^{f)}$ Combined results of refs. [35,36].

[37]. For $\mathrm{Be}$ and $\mathrm{Ne}$, the accurate $\mathrm{CI}$ results of Esquivel and Bunge were used [33,34].

We first discuss the merits of the exchange-only potentials $\mathrm{KS}_{\mathrm{X}}, \mathrm{LM}_{\mathrm{X}}$, and $\mathrm{B}_{\mathrm{X}}$. In figs. 1a, $2 \mathrm{a}$ and $3 \mathrm{a}$, the relative difference between the electron density obtained with these potentials and the HF density is depicted for $\mathrm{He}$, Be, and $\mathrm{Ne}$, respectively. From these results, together with those summarized in table 1 , we infer that the non-local exchange-only potentials $\mathrm{LM}_{\mathrm{X}}$ and $\mathrm{B}_{\mathrm{X}}$ represent a significant, though slight, improved on the local $\mathrm{KS}_{\mathrm{X}}$ potential. Whereas the electron density obtained with the local potential is systematically too low in the high-density region, the non-local $B_{x}$ results are generally too high. Both the non-local potentials diverge to minus infinity at the nuclear cusps. Although this divergence, originating from the singular character of $\nabla^{2} \rho$ at the nuclear cusp, reportedly presents no serious problem for the ex- change energy, it has a non-negligible influence on the electron density in the core region. The potential $\mathrm{LM}_{\mathrm{X}}$ almost completely compensates for the deficiencies of the local potential at the nuclear cusp. The leading term in the potential close to the nuclear cusp is $0.0051\left(\nabla^{2} \rho\right) / \rho^{4 / 3}$, compared to $0.0091\left(\nabla^{2} \rho\right) /$ $\rho^{4 / 3}$ in the case of potential $B_{X}$. Regarding both potentials as a non-local correction to the local potentials $\mathrm{KS}_{\mathrm{X}}$ explains why the difference $\rho_{\mathrm{BX}}(0)-$ $\rho_{\mathrm{KSx}}(0)$ is almost twice the difference $\rho_{\mathrm{LMx}}(0)-$ $\rho_{\mathrm{KSx}}(0)$. It can be concluded that both non-local potentials $L_{M_{X}}$ and $B_{X}$ can be regarded as an improvement on the local approximation to exchange, in that they compensate for some deficiencies of the latter, namely a too low core density and too high valence density. Potential $\mathbf{B}_{\mathbf{X}}$, however, overcompensates for these deficiencies.

Including correlation by adding the local correla- 


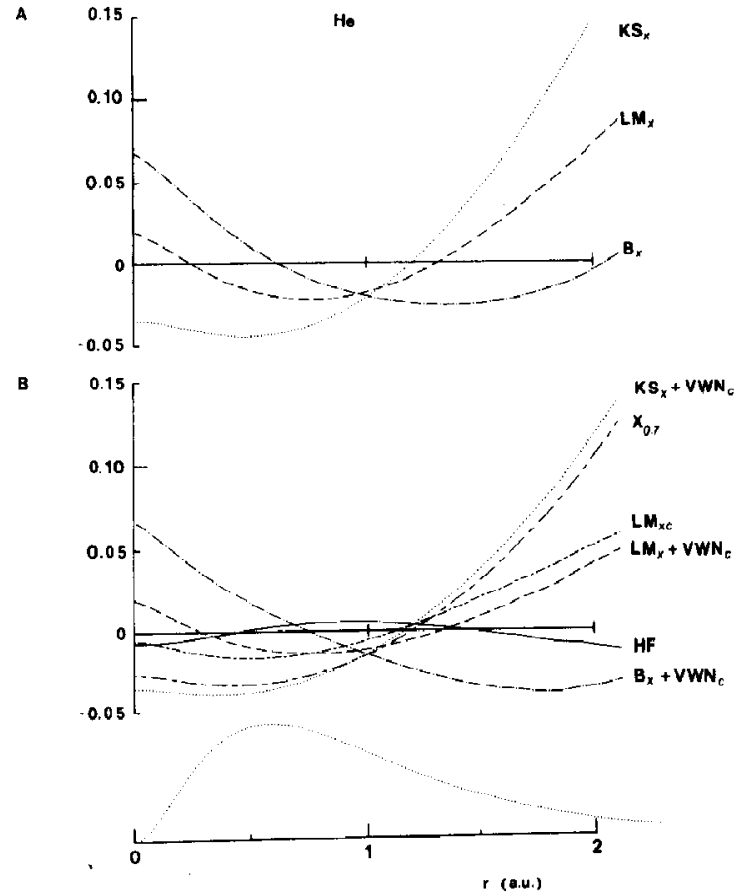

Fig. 1. Relative deviation from the HF and exact results for the electron density in $\mathrm{He}$, obtained for various exchange-correlation potentials. The radial electron density $4 \pi r^{2} \rho$ is given for comparison. (a) $\left(\rho-\rho_{\mathrm{HF}}\right) / \rho_{\mathrm{HF}}$. (b) $\left(\rho-\rho_{\text {Exact }}\right) / \rho_{\text {Exact }}$.

tion potential $\mathrm{VWN}_{\mathrm{C}}$, corrected for self-correlation, does not markedly improve the agreement with the exact results, as can be readily inferred from table 1 . The errors in the exchange-only results are in general larger than and not compensated by the changes induced by including correlation within a local approximation. The relative errors between results obtained this way and exact results, as depicted in figs. $1 \mathrm{~b}, 2 \mathrm{~b}$, and $3 \mathrm{~b}$ for $\mathrm{He}, \mathrm{Be}$, and $\mathrm{Ne}$, respectively, support this conclusion. The $\mathrm{X} \alpha$ results, with $\alpha=0.7$, do not differ significantly from results obtained with the local $\mathrm{KS}_{\mathrm{X}}$ exchange-only potential augmented with the local correlation-only potential $\mathrm{VWN}_{\mathrm{C}}$, corrected for self-correlation. Similarly, results obtained with the non-local exchange-correlation potential $\mathrm{LM}_{\mathbf{x C}}$ do not differ significantly from those obtained with the non-local exchange-only potential $\mathrm{LM}_{\mathrm{X}}$ augmented with the self-correlation corrected local expression for correlation-only, $\mathrm{VWN}_{\mathrm{C}}$.

We conclude that, although results obtained with the non-local exchange-correlation potentials de-

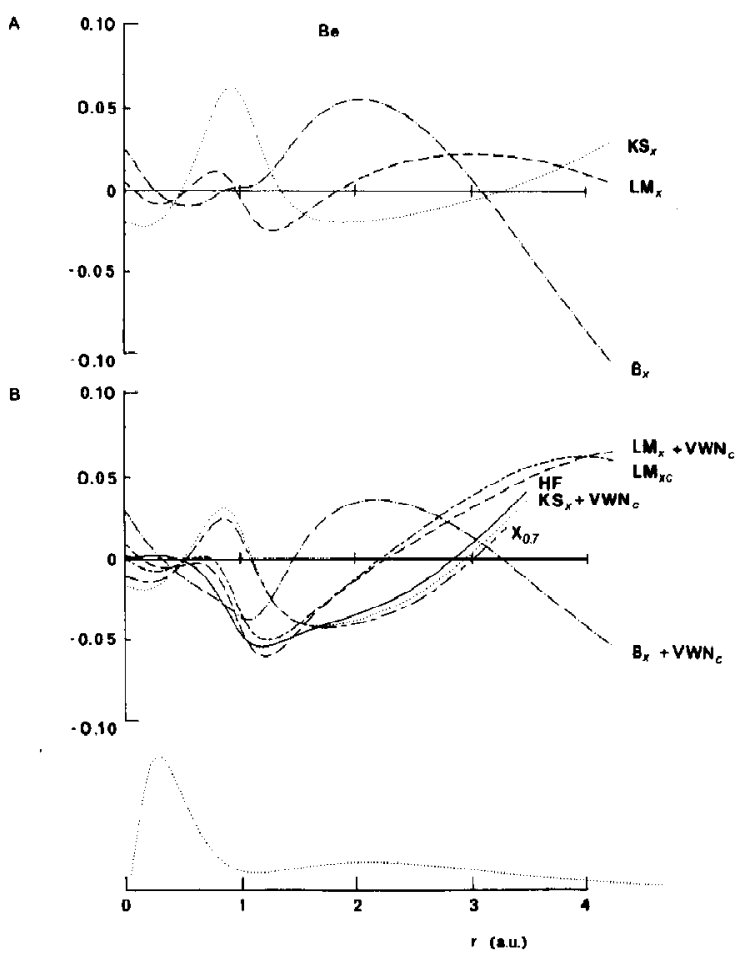

Fig. 2. Relative deviation from the HF and exact results for the elcetron density in $\mathrm{Be}$, obtained for various exchange-correlation potentials. The radial electron density $4 \pi r^{2} \rho$ is given for comparison. (a) $\left(\rho-\rho_{\mathrm{HF}}\right) / \rho_{\mathrm{HF} .}$ (b) $\left(\rho-\rho_{\text {Exact }}\right) / \rho_{\text {Exact }}$.

scribed above are superior to those obtained with local potentials, the largest discrepancies with the exact results are still mainly due to an improper description of the exchange part of the exchange-correlation potential.

Next we focus on the density response to the correlation potential, i.e. the difference between the electron density calculated self-consistently with and without correlation. In fig. 4 , the exact density response, i.e. the difference between the exact and the HF density, is compared with the density response to the local approximation $\mathrm{VWN}_{\mathrm{C}}$, corrected for selfcorrelation, and also the response to a non-local correlation-only expression suggested by Langreth and Mehl [12], hereafter denoted as $\mathbf{L M}_{\mathbf{C}}$. In contrast to results not corrected for self-correlation (see e.g. fig. 8 in ref. [27]), the effect of eliminating the local correlation potential $\mathrm{VWN}_{\mathrm{C}}$ for self-correlation, according to the method of Stoll et al. [17,18], is to reduce the density response to the correlation potential by approximately a factor of two, thereby im- 


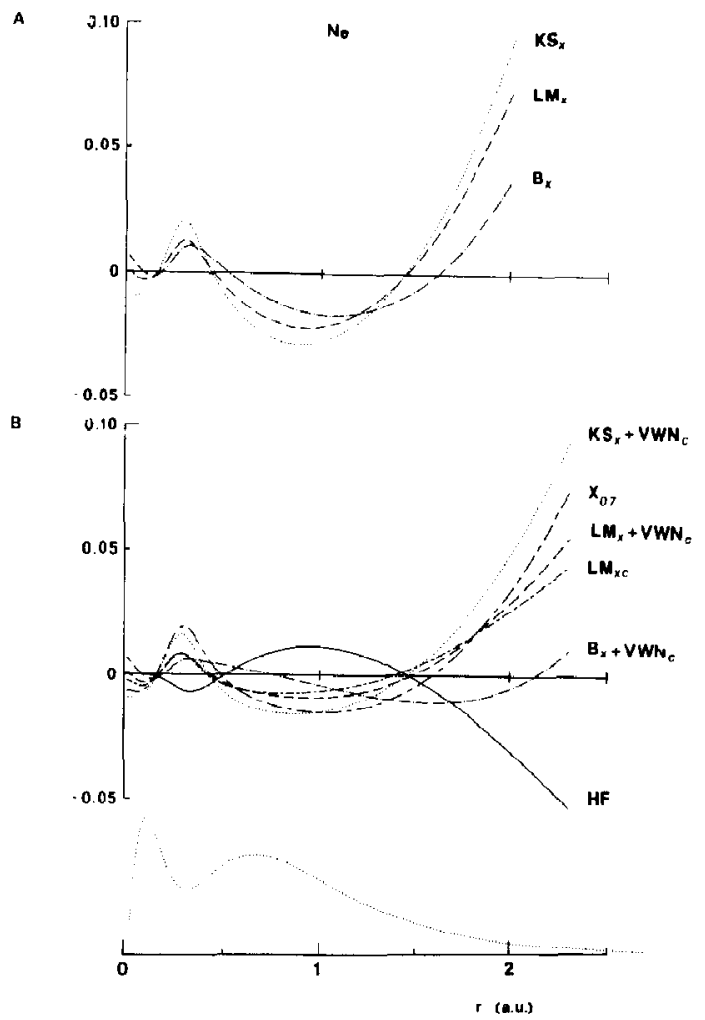

Fig. 3. Relative deviation from the HF and exact results for the electron density in $\mathrm{Ne}$, obtained for various exchange-correlation potentials. The radial electron density $4 \pi r^{2} \rho$ is given for comparison. (a) $\left(\rho-\rho_{\mathrm{HF}}\right) / \rho_{\mathrm{HF} .}$ (b) $\left(\rho-\rho_{\text {Exact }}\right) / \rho_{\text {Exact }}$

proving the agreement with the exact results for He. The density response to the correlation potential appears to be virtually independent of the exchange potential used. For He, the tendency of correlation to reduce the density in the intermediate region cannot be reproduced by the local or non-local correlation potential. Close to the nuclear cusp, the non-local approximation fails. Both local and non-local correlation potentials poorly represent the exact density response for $\mathrm{Be}$ and $\mathrm{Ne}$. Although one expects a local correlation potential to work well in the valence region, this is obviously not the case. It must be kept in mind, however, that since the total number of electrons is conserved, an erroneous response of the density in the core region must be compensated for by an equally erroneous response in the valence region. The non-local potential $\mathrm{LM}_{\mathrm{C}}$ cannot be regarded as an improvement on the local potential $\mathrm{VWN}_{\mathrm{C}}$, corrected for self-correlation.

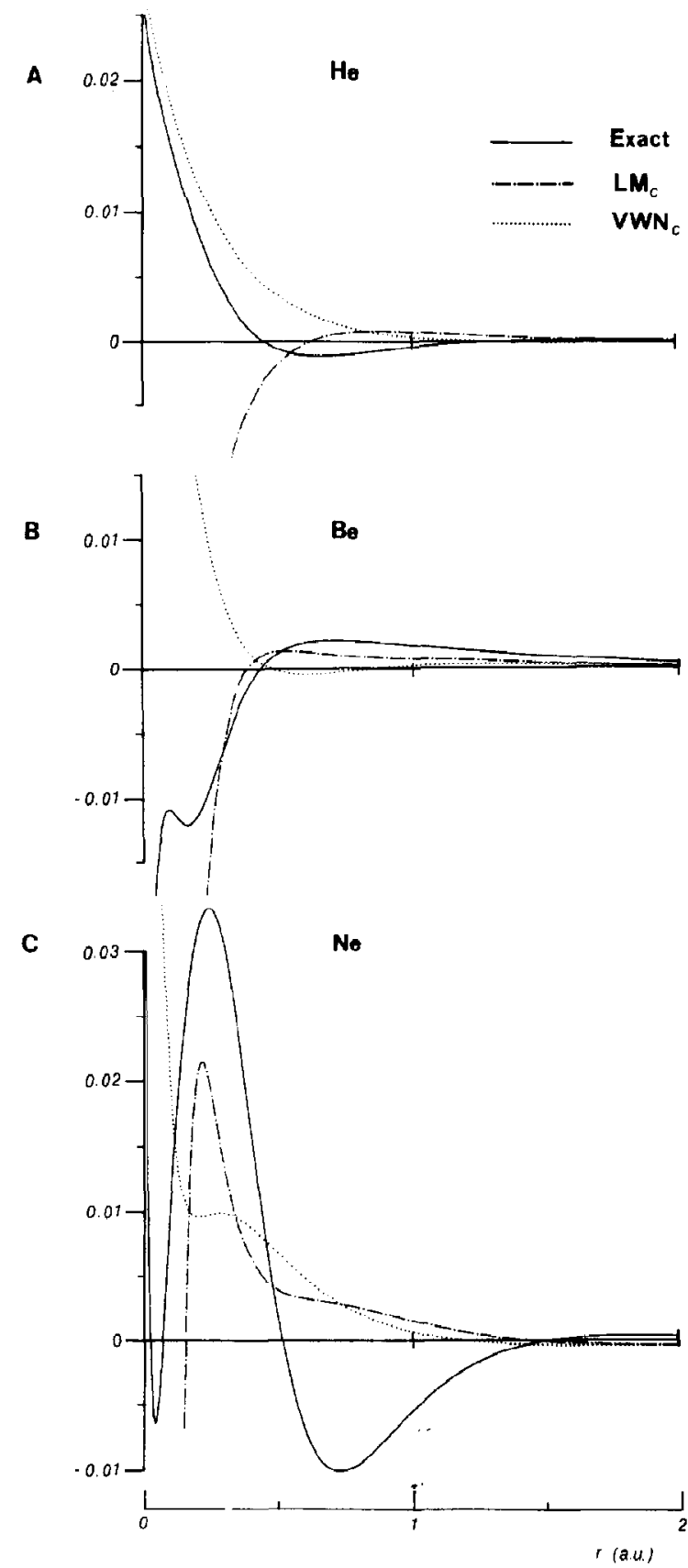

Fig. 4. Response of the electron density following inclusion of correlation. (a) He. (b) Be. (c) Ne.

For the highest occupied orbital eigenvalues, as summarized in table 1 , the non-local exchange-correlation potentials discussed above do not represent a significant improvement on their local counterparts.

In summary, although the non-local exchange- 
correlation energy functionals discussed above reportedly give results for exchange-correlation energies an order of magnitude better than their local counterparts, the electron density distribution, obtained self-consistently with exchange-correlation potentials obtained as their functional derivative, is only moderately improved.

\subsection{Molecules}

Geometries and spectroscopic properties of molecules are surprisingly accurately predicted by density functional methods, and are often superior to $\mathrm{HF}$ results. For example, to obtain the correct dissociation limit for $\mathbf{H}_{2}$, correlation corrections must be applied to HF theory, whereas local approximations to the exchange-correlation potential result in a correct dissociation into neutral atoms. Even local exchange-only potentials, thus neglecting correlation, predict the proper dissociation limit. This puzzle has been largely resolved recently by Cook and Karplus [45], who show that in molecules, within the singledeterminant HF approximation, the presence of stateand geometry-dependent ionic errors, relative to the correct HF description of the individual free atoms constituting the molecule, are responsible for most of the error in the HF potential surface, especially at large bond distances. These errors disappear on squaring the single-determinant wavefunction, and are thus not present in the electron density, from which the exchange (-correlation) potential is calculated in DFT. From this we infer that, for atoms, considering the results of the previous section, results for the electron density obtained from DFT calculations with a local approximation to exchange (and correlation) will, in general show little improvement over HF results. However, for molecular systems, DFT will give a better description of the deformation density, i.e. the density redistribution taking place upon forming the molecule (or solid) out of the free atoms constituting the molecule (or solid). We therefore expect estimates of dipole moments obtained from DFT to be in closer agreement with CI results or experiment than the corresponding HF results. There is evidence that this is indeed the case. In table 2 , values for the dipole monnent of the ground states of $\mathrm{CO}\left({ }^{1} \Sigma^{+}\right)$and $\mathrm{H}_{2} \mathrm{O}\left({ }^{1} \mathrm{~A}_{1}\right)$ are listed for various exchange-correlation potentials. In all cases,
Table 2

Dipole moments (atomic units) for $\mathrm{CO}$ and $\mathrm{H}_{2} \mathrm{O}$, obtained for various exchange-correlation potentiàls

\begin{tabular}{lll}
\hline & $\mathrm{CO}$ & $\mathrm{H}_{2} \mathrm{O}$ \\
\hline $\mathrm{X} 0.7$ & -0.096 & 0.743 \\
& $-0.095^{\text {a) }}$ & \\
$\mathrm{KS}_{\mathrm{X}}$ & -0.111 & 0.731 \\
$\mathrm{KS}_{\mathrm{X}}+\mathrm{VWN}_{\mathrm{C}}$ & -0.103 & 0.741 \\
$\mathrm{LM}_{\mathrm{Xc}}$ & -0.080 & \\
$\mathrm{HF}$ & $0.104^{\mathrm{b}}$ & $0.787^{\mathrm{e}}$ \\
$\mathrm{CI}$ & $-0.126^{\mathrm{c}}$ & $0.759^{\mathrm{f}}$ \\
exp. & $-0.048^{\text {d) }}$ & $0.730^{\mathrm{s})}$ \\
\hline
\end{tabular}

a) Fully numerical result of Laaksonen et al. [38 ].

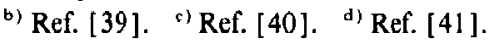

') Ref. [42]. ') Ref. [43]. ") Ref. [44].

density functional results are superior to HF results, and predict the proper sign for the dipole moment of $\mathrm{CO}$. Equilibrium geometries for $\mathrm{CO}$ and $\mathrm{H}_{2} \mathrm{O}$ were taken from ref. [46] and ref. [47], respectively. Density gradients, present in the expression for the non-local exchange-correlation potential $\mathrm{LM}_{\mathrm{Xc}}$, were calculated analytically from the density $\tilde{\rho}$ in eq. (9).

The deformation density, obtained by subtracting the superposition of spherically averaged atomic densities from the molecular electron density, provides direct information about the various aspects of chemical bonding. In the case of $\mathrm{CO}$ the deformation density does not seem to be very dependent on the choice of exchange-correlation potential. The difference between the non-local $\mathrm{LM}_{\mathrm{XC}}$ and local X0.7 and $\mathrm{KS}_{\mathrm{X}}+\mathrm{VWN}_{\mathrm{C}}$ results amounts to $2.5 \%$ at the midpoint of the bond, whereas, from a previous calculation [23], we inferred that omitting f-type polarization functions diminishes the deformation density by more than $10 \%$ at the bond midpoint. Hence errors due to basis set inadequacies do, in general, outweigh errors introduced by approximating the exchange-correlation potential with a local expression. It should be mentioned, however, that the above arguments only hold provided the reference state, i.e. the free atoms constituting the molecule, is calculated with the same basis set and exchange-correlation potentials as used for the molecule, since only then can inadequacies in the basis set and exchange-conrelation potential be expected to cancel.

Finally we consider the net effect on the electron 

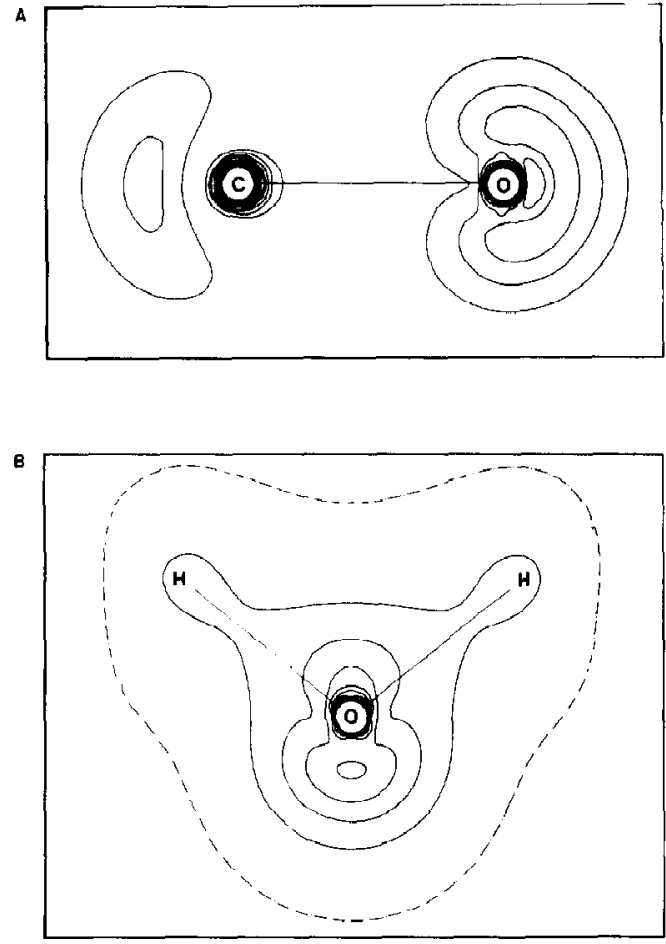

Fig. 5. Response of the electron density following inclusion of correlation. Shown is $\Delta \rho=\rho_{\mathrm{KS}}+\mathrm{VwNC}-\rho_{\mathrm{KS}}$. Solid lines denote electron excess, dash-dotted lines are zero contours. The contour interval is $0.01 e \AA^{-3}\left(1 e a_{0}^{-3} \approx 6.748 e \AA^{-3}\right)$. (a) $\mathrm{CO}$. (b) $\mathrm{H}_{2} \mathrm{O}$.

density of taking into account correlation within the local density approximation. The response of the electron density to the addition of the correlation potential $\mathrm{VWN}_{\mathrm{C}}$ to the exchange-only potential $\mathrm{KS}_{\mathrm{X}}$ is depicted in figs. $5 \mathrm{a}$ and $5 \mathrm{~b}$ for $\mathrm{CO}$ and $\mathrm{H}_{2} \mathrm{O}$ respectively. Comparing these results with those obtained from CI calculations [48-50], only the order of magnitude agrees. There is complete disagreement between the topological structure of the DFT and CI results.

\section{Conclusions}

A systematic comparison of essentially exact electron density distributions with those obtained for various local and non-local approximations to the exchangc-correlation potential, lcads us to the following conclusions:

(i) Although non-local functionals give results for exchange and correlation energies an order of magnitude better than their local counterparts, electron densities, calculated self-consistently with an exchange-correlation potential obtained as a functional derivative of the corresponding energy functional, are only modestly improved. A similar conclusion has been reached by Pedroza [51].

(ii) Errors in the electron density, due to inadequacies in local and non-local approximations to the exchange part of the exchange-correlation potential, outweigh the correlation contribution to the electron density. Therefore, efforts to improve on present local and non-local exchange-correlation potentials should focus on the exchange part.

(iii) The response of the electron density to correlation is only qualitatively reproduced by the local and non-local correlation potentials discussed in the present paper.

(iv) There is evidence that density functional methods yield deformation densities in better agreement with $\mathrm{CI}$ results or experiment than $\mathrm{HF}$ results.

\section{References}

[1] P. Becker, ed., Electron and magnetization densities in molecules and crystals (Plenum Press, New York, 1980).

[2] P. Coppens and M.B. Hall, eds., Electron distributions and the chemical bond (Plenum Press, New York, 1982).

[3] A.S. Bamzai and B.M. Deb, Rev. Mod. Phys. 53 (1981) 95.

[4] P. Hohenberg and W. Kohn, Phys. Rev. 136 (1964) B864.

[5] J. Callaway and N.H. March, Solid State Phys. 38 (1984) 135.

[6] W. Kohn and L.J. Sham, Phys. Rev. 140 (1965) Al133.

[7] O. Gunnarsson, B.I. Lundqvist and J.W. Wilkins, Phys. Rev. B 10 (1974) 1319.

[8] O. Gunnarsson and B.I. Lundqvist, Phys. Rev. B 13 (1976) 4274.

[9] O. Gunnarsson, M. Jonson and B.I. Lundqvist, Phys. Rev. B 20 (1979) 3136

[10] D.C. Langreth and J.P. Perdew, Phys. Rev. B 15 (1977) 2884.

[11] D.C. Langreth and J.P. Perdew, Phys. Rev. B 21 (1980) 5469.

[12] D.C. Langreth and M.J. Mehl, Phys. Rev. B 28 (1983) 1809.

[13] C.D. Hu and D.C. Langreth, Phys. Rev. B 33 (1986) 943.

[14] S.H. Vosko, L. Wilk and M. Nusair, Can. J. Phys. 58 (1980) 1200.

[15] S.H. Vosko and L. Wilk, Phys. Rev. B 22 (1980) 3812.

[16] D.M. Ceperley and B.J. Alder, Phys. Rev. Letters 45 (1980) 566. 
[17] H. Stoll, C.M.E. Pavlidou and H. Preuss, Theoret. Chim. Acta 49 (1978) 143.

[18] H. Stoll, E. Golka and H. Preuss, Theoret. Chim. Acta 55 (1980) 29.

[19] D.E. Ellis and G.S. Painter, Phys. Rev. B 2 (1970) 2887.

[20] E.J. Baerends, D.E. Ellis and P. Ros, Chem. Phys. 2 (1973) 41.

[21] E.J. Baerends and P. Ros, Chem. Phys. 2 (1973) 52.

[22] P.M. Boerrigter, Ph.D. Dissertation, Free University of Amsterdam (1987).

[23] E.J. Baerends, P. Vernooijs, P.M. Boerrigter, M.P.C.M. Krijn, D. Feil and D. Sundholm, J. Mol. Struct. 133 (1985) 147.

[24] M.P.C.M. Krijn and D. Feil, to be published.

[25] O. Gunnarsson and R.O. Jones, Physica Scripta 21 (1980) 394.

[26] G.S. Painter, Phys. Rev. B 24 (1981) 4264.

[27] J.P. Perdew and A. Zunger, Phys. Rev. B 23 (1981) 5048.

[28] A.D. Becke, J. Chem. Phys. 84 (1986) 4524.

[29] C.O. Almbladh and U. von Barth, Phys. Rev. B 31 (1985) 3231.

[30] C. Froese Fischer, The Hartree-Fock method for atoms (Wiley, Ncw York, 1977).

[31] E. Clementi and C. Roetti, At. Data Nucl. Data Tables 14 (1974) 177.

[32] C.-O. Almbladh, U. Ekenberg and A.C. Pedroza, Physica Scripta 28 (1983) 389.

[33] R.O. Esquivel and A.V. Intern. J. Quantum Chem. 32 (1987) 295.
[34] A.V. Bunge and R.O. Esquivel, Phys. Rev. A 34 (1986) 853.

[35] C.-O. Almbladh and A.C. Pedroza, Phys. Rev. A 29 (1984) 2322.

[36] K. Schwartz, J. Phys. B 11 (1978) 1339.

[37] C.-O. Almbladh and S. Larsson, private communication.

[38] L. Laaksonen, D. Sundholm and P. Pyykkö, Intern. J. Quantum Chem. 27 (1985) 601.

[39] P.A. Christiansen and E.A. McCullough, J. Chem. Phys. 67 (1977) 1877.

[40] K. Kirkby-Docken and B. Liu, J. Chem. Phys. 66 (1977) 4309.

[41] C. Chackerian, J. Chem. Phys. 65 (1976) 4228.

[42] J.G.C.M. van Duijneveldt-van de Rijdt and F.B. van Duijneveldt, J. Mol. Struct. 89 (1982) 185.

[43] E.R. Davidson and D. Feller, Chem. Phys. Lettcrs 104 (1984) 54.

[44] T.R. Dyke and J.S. Muenter, J. Chem. Phys. 59 (1973) 3125.

[45] M. Cook and M. Karplus, J. Phys. Chem. 91 (1987) 31.

[46] K.P. Huber, in: American Institute of Physics handbook, ed. D.E. Gray (McGraw-Hill, New York, 1972).

[47] W.S. Benedict, N. Gailar and E.K. Plyler, J. Chem. Phys. 24 (1956) 1139.

[48] V.H. Smith, Physica Scripta 15 (1977) 147.

[49] H. Meyer, A.Schweig and W. Zittlau, Chem. Phys. Letters 92 (1982) 637.

[50] M. Breitenstein, H. Dannöhl, H. Meyer, A. Schweig, R. Seeger, U. Seeger and W. Zittlau, Intern. Rev. Phys. Chem. 3 (1983) 335.

[51] A.C. Pedroza, Phys. Rev. A 33 (1986) 804. 